\title{
Relación fuente-destino en cultivares de maní tipo runner (Arachis hypogaea L.) sembrados en Argentina
}

\author{
Morla, F. D., Giayetto, O., Fernandez, E. M. y Cerioni, G. A.
}

\begin{abstract}
RESUMEN
La partición de asimilados a frutos es una característica fisiológica de relevancia para el aumento del rendimiento de maní, y su análisis mediante la relación fuente-destino aporta al mejoramiento genético y manejo del cultivo. El objetivo fue analizar la relación fuente-destino en cultivares runner sembrados en Argentina. Se realizaron dos experimentos a campo: en el Exp1 el cultivar Granoleico fue sembrado en tres fechas durante 2009-2010 y 2010-2011. En el Exp2 fueron sembrados seis cultivares runner (Florunner, Florman, Manigran, Asem-485, Pepe-Asem y Granoleico) durante 2011-2012. La relación fuentedestino se analizó con dos metodologías: la biomasa total asignada a cada fruto durante su llenado con relación a su peso final, y el análisis de la relación entre peso y número de frutos a cosecha. La falta de compensación entre número y peso de frutos demostró que el maní podría llenar un amplio número de frutos (entre 20 y 57 frutos planta-1 ${ }^{-1}$. Además, el peso medio de un fruto $(1,05 \mathrm{~g})$ fue menor al crecimiento total de la planta asignado a él durante su período de formación (2,63 g). Se determinó una limitación por destinos, indicando la posibilidad de aumentar el rendimiento a través del incremento del tamaño de los destinos.
\end{abstract}

Palabras clave: maní; relación fuente-destino; cultivares tipo runner; fechas de siembra.

Morla, F. D., Giayetto, O., Fernández, E. M. and Cerioni, G. A. 2019. Source-Sink relationship in runner-type peanut cultivars (Arachis hypogaea L.) grown in Argentina. Agriscientia 36 (2): 39-44

\section{SUMMARY}

Assimilate partitioning into reproductive structures is a relevant physiological feature in increasing peanut yield, and its analysis through the source-sink relationship is an important contribution to genetic improvement and crop management. The objective was to analyze the source-sink relationship of runner-type cultivars grown in Argentina. Two field experiments were performed, Exp1 consisted in the analysis of the cultivar Granoleico in three sowing 
dates during 2009-2010 and 2010-2011. In Exp2, six runner-type cultivars (Florunner, Florman, Manigran, Asem-485, Pepe-Asem and Granoleico) were sown during 2011-2012. The source-sink relationship was analyzed using two methodologies: total biomass assigned to each pod during pod filling period $\left(\mathrm{g} \mathrm{pod}^{-1}\right)$ in relation to its final weight, and analysis of the trade-off between pod number and pod weight at harvest. The lack of trade-off between pod number and weight showed that the peanut plant has conditions to fill a wide number of pods (20-57 pods plant $\left.{ }^{-1}\right)$ in the same way. Also, the average pod weight $(1.05 \mathrm{~g})$ was lower than the total plant biomass assigned to that pod during its formation $(2.63 \mathrm{~g})$. A marked limitation by sinks was determined, indicating the possibility of to increase the peanut yield by means of improvements in sinks size.

Key words: peanut; source-sink relationship; runner-type cultivars; sowing dates.

Morla F. D., Giayetto, O., Fernandez, E. M. y Cerioni, G. Á. Universidad Nacional de Río Cuarto, Facultad de Agronomía y Veterinaria, Departamento de Producción Vegetal. X5804BYA, Ruta N. 36, Km 601, Río Cuarto, Córdoba, Argentina. Correspondencia a: fmorla@ayv.unrc.edu.ar

\section{INTRODUCCIÓN}

El rendimiento de un cultivo depende de la capacidad de acumular biomasa en los órganos cosechables, por lo que un incremento de la biomasa destinada a tales órganos aseguraría un mayor rendimiento (Egli y Bruening, 2001). Esta distribución de materia seca entre los diferentes órganos de la planta es el resultado final de un conjunto ordenado de procesos metabólicos y de transporte, que gobiernan el flujo de asimilados a través de un sistema fuente-destino (Gifford y Evans, 1981).

Duncan, McCloud, McGraw y Boote (1978) definieron la partición de asimilados hacia las estructuras reproductivas, o índice de cosecha, como una característica fisiológica de relevancia en el aumento del rendimiento del cultivo de maní (Arachis hypogaea L.). En términos de regulación, la producción y el llenado de granos pueden ser limitados por la disponibilidad o la utilización de esos asimilados; es decir, limitación por fuente o destino, respectivamente (Gifford y Evans, 1981).

El maní se caracteriza por tener un crecimiento marcadamente indeterminado, con crecimiento vegetativo puro solamente en una etapa inicial muy corta de su ciclo (Stalker, 1997). Cumplida esa fase, florece y luego los frutos inician su desarrollo, convirtiéndose progresivamente en los principales órganos destino. Con la aparición paulatina de nuevos frutos, se inicia una competencia por los asimilados disponibles entre ellos y los órganos vegetativos (hojas, tallos y raíces) aún en activo crecimiento (Huyghe, 1998).

Uno de los importantes avances de la ecofisiología de cultivos ha sido aumentar la comprensión de la generación del rendimiento y sus componentes numéricos (número y peso de granos), mediante la manipulación de la disponibilidad de asimilados durante diferentes etapas fenológicas de los cultivos (Fischer, 1975, en trigo; Egli y Bruening, 2001, en soja; Cantagallo, Medan y Hall, 2004, en girasol; Gambín, Borrás y Otegui, 2006, y Cerrudo et al., 2013, en maíz).

En el caso del maní también existen numerosos trabajos en los que se modificó la relación fuentedestino a través de: sombreo (Hang, McCloud, Boote y Duncan, 1984); raleo de plantas (Williams, 1979); defoliación y remoción de frutos (Ghosh y Biswas, 1995); control de la floración y formación de los frutos (Hemsy, Rodriguez, Scandaliaris, Lozano Muñoz y Cajal, 1974; Ghosh y Biswas, 1995); y control del clavado (Haro, Dardanelli, Otegui y Collino, 2008; Haro, Dardanelli, Collino y Otegui, 2010).

Por otro lado, hay diferentes metodologías para la determinación de la relación fuente-destino. Gambín y Borrás (2010) utilizaron el modelo teórico de Smith y Fretwell (1974) para comprender las relaciones entre el número de granos, el peso del grano y los recursos disponibles de quince especies cultivadas. Este modelo se basa en el conocimiento del efecto de compensación en el que una cantidad fija de los recursos disponibles se puede distribuir en diferentes combinaciones de números y peso de granos en la planta. Así, en 
los recursos disponibles las variaciones debidas al genotipo y el medio ambiente pueden resultar en relaciones negativas, neutras o positivas entre el número y el peso de los granos en plantas individuales (Venable, 1992).

También se sabe que cambios en la producción de asimilados en la fuente afectan directamente su disponibilidad para el desarrollo de los destinos, por lo tanto, las diferencias en el crecimiento de la planta por grano (medido como el aumento de biomasa aérea total por grano fijado) durante el período de su formación, implican distintas cantidades de asimilados disponibles (relación fuente destino, RFD) para el desarrollo de los granos (Gambín y Borrás, 2010).

En maní se encontraron distintos resultados al analizar fechas de siembra (Haro, Otegui, Collino y Dardanelli, 2007), genotipos (Haro, Baldessari y Otegui, 2013) y ambientes (Nautiyal, Ravindra, Rathnakumar, Ajay y Zala, 2012). En los citados trabajos se describe a la especie, principalmente, limitada por destinos.

El objetivo del presente trabajo fue analizar, con diferentes metodologías de estudio, la variabilidad de la relación fuente-destino en diferentes genotipos de maní tipo runner utilizados en Argentina.

\section{MATERIALES Y MÉTODOS}

El experimento 1 (Exp1) consistió en ensayos realizados con el cultivar Granoleico (de tipo runner, ampliamente sembrado en la región manisera argentina) sembrado en una fecha de siembra temprana ( $\left.1^{\circ} \mathrm{FS}\right)$, intermedia $\left(2^{\circ} \mathrm{FS}\right)$ y tardía $\left(3^{\circ} \mathrm{FS}\right)$, en un diseño de bloques completos aleatorizados durante los ciclos experimentales 2009-2010 y 2010-2011 (ver detalles en Morla, Giayetto, Cerioni y Fernandez, 2016).

El experimento 2 (Exp2) se basó en la comparación de seis cultivares tipo runner: Florunner, Florman, Manigran, Asem 485, Pepe Asem y Granoleico sembrados el 10/11/2011, dispuestos en un diseño completamente aleatorizado con tres repeticiones y un espaciamiento de 0,70 $\mathrm{m}$ entre surcos y de 0,08 m entre plantas.

En ambos experimentos el cultivo se condujo sin limitaciones hídricas ni nutricionales, y se aplicó un manejo sanitario con el objeto de mantenerlo libre del efecto de malezas, plagas y enfermedades. El análisis de la relación fuente-destino se realizó mediante dos metodologías empleadas en el cultivo de maní y en otros cultivos.

Durante el período de definición de los componentes del rendimiento se aplicó la metodología usada por Haro et al. (2007) y Haro et al. (2013) en maní, y por Gambín et al. (2006) en maíz. Esta relación fue estimada como el crecimiento total del cultivo por cada fruto durante el período de formación de ese fruto (RFD, en $\mathrm{g}$ fruto- $^{-1}$ ), establecido entre las etapas fenológicas R3-4 y R8 (Giayetto et al., 2013). La etapa fenológica R8, o madurez de arrancado, fue modificada de Boote (1982) y se determinó según el porcentaje de frutos maduros, $45 \%$. El cálculo consistió en el cociente entre la producción total de biomasa en esa etapa y el número de frutos maduros (según metodología de Williams y Drexler, 1981) obtenidos al momento de la cosecha.

RFD ( $\mathrm{g}$ fruto $\left.^{-1}\right)=\underline{\Delta \text { Biomasa en el Período de llenado del fruto (R3/4-R8) }}$ Número de frutos maduros a cosecha

El peso final de un fruto maduro puede ser: i) mayor que la RFD, indicando el uso de reservas y una limitación por fuente; ii) menor que la RFD, indicando una oferta mayor que la demanda y una limitación por destinos; o iii) de un valor similar a la RFD, que sugiere una equivalencia entre la oferta y la demanda (Haro et al., 2013).

La otra metodología de análisis fue mediante la relación entre el peso y el número de frutos cosechables. Esta ha sido utilizada por Gambín y Borrás (2010) en diferentes cultivos, por Haro et al. (2013) en maní, y en maíz por Cerrudo et al. (2013). La metodología considera que, si los resultados muestran un efecto de compensación (i.e. los puntos se mantienen dentro de líneas de isocuantas de rendimiento), existe una colimitación por fuente y destino; mientras que la falta de compensación entre estos componentes estaría indicando una limitación por fuente (pendientes de la regresión altamente negativas) o por destinos (pendientes de la regresión cercanas a cero o positivas).

Las variables bajo estudio se sometieron a un análisis de regresión y correlación utilizando el programa estadístico GraphPad Prism versión 5.00 para Windows.

\section{RESULTADOS Y DISCUSIÓN}

El cultivar Granoleico, evaluado en el Exp1, no presentó efecto de compensación entre los componentes directos del rendimiento, peso individual en función del número de frutos maduros por planta (Figura 1). Los pares de datos exploraron las isocuantas de rendimiento de 20 a $50 \mathrm{~g} \mathrm{pl}^{-1}$, y ajustaron a una regresión lineal para las tres fechas de siembra y los dos ciclos experimentales 
evaluados $(y=-0,0007 x+1,15)$. El número de frutos maduros varió en un rango de 18 a 47 frutos $\mathrm{pl}^{-1} \mathrm{y}$ el peso individual promedio de estos frutos fue de $1,13 \mathrm{~g}$.

En el Exp2, y para los diferentes genotipos, se observó un patrón de distribución similar a los descriptos en el Exp1 (Figura 2). Los cultivares evaluados presentaron rendimientos de frutos en un rango de 14,2 (Florman INTA) a 60,9 $\mathrm{g} \mathrm{pl}^{-1}$ (Granoleico); los pares de datos ajustaron a un modelo lineal: $y=-0,0012 x+1,02$.

El peso medio de un fruto maduro fue de 0,98 9 y el número de frutos maduros varió entre 20 y 57 frutos $\mathrm{pl}^{-1}$.

La falta de compensación (trade-off) entre los principales componentes del rendimiento, número y peso de frutos, en los escenarios analizados demuestra que la planta de maní está en condiciones de llenar, de la misma manera, un amplio rango de número de frutos. Esta repuesta se observó de manera similar para diferentes genotipos (Figura 2), fechas de siembra y ciclos experimentales (Figura 1). Resultados análogos a estos fueron obtenidos por Haro et al. (2013) quienes no encontraron limitación por fuente en cuatro cultivares tipo Valencia y Español y otros cuatro tipo runner.

Este tipo de respuesta indica un exceso de fuente (aparato foliar) durante el período de formación de los frutos. En este sentido, Ghosh y Biswas (1995) describen que una defoliación del $25 \%$ no modificó el rendimiento del cultivo (solo se produjo una disminución del $2 \%$ del rendimiento en granos) y las plantas no presentaron síntomas de limitación por fuente (senescencia temprana).

El peso medio de un fruto maduro fue marcadamente menor al crecimiento total de la biomasa de la planta asignada a ese fruto durante su período de formación $\left(\mathrm{RFD}_{\mathrm{R} 3 / 4-\mathrm{R} 8}\right)$, para las tres fechas de siembra y los dos ciclos experimentales analizados en este trabajo (Figura 3). La RFD varió entre 1,7 ( $3^{\circ} \mathrm{FS} 2009-2010$ ) y 2,63 $\mathrm{g}_{\text {fruto- }}{ }^{-1}$ ( $2^{\circ}$ FS 2010-2011) con un promedio de los datos analizados de 2,13 g; mientras que el peso medio de un fruto fue de $1,05 \mathrm{~g}$, y varió entre 0,81 y 1,28 g.

Estos resultados indican el potencial que todavía queda por explorar en el mejoramiento de maní. Similares resultados fueron descriptos por Haro et al. (2013), quienes remarcan que la alta relación fuente-destino encontrada en maní para la fase de llenado del grano (posterior a la fase R5), sugeriría que no existen limitaciones para seguir mejorando por un mayor número de frutos (o granos), formados (aumento en la eficiencia

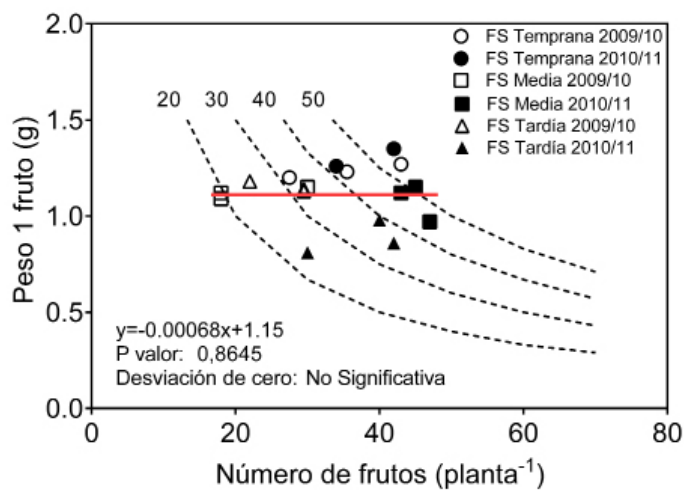

Figura 1. Peso individual de un fruto (g) en función del número de frutos maduros por planta (frutos $\mathrm{pl}^{-1}$ ) para el cultivar Granoleico sembrado en tres fechas de siembra y dos ciclos experimentales y su regresión lineal (línea continua). Las líneas punteadas representan isocuantas de rendimiento $\left(\mathrm{g} \mathrm{pl}^{-1}\right)$.

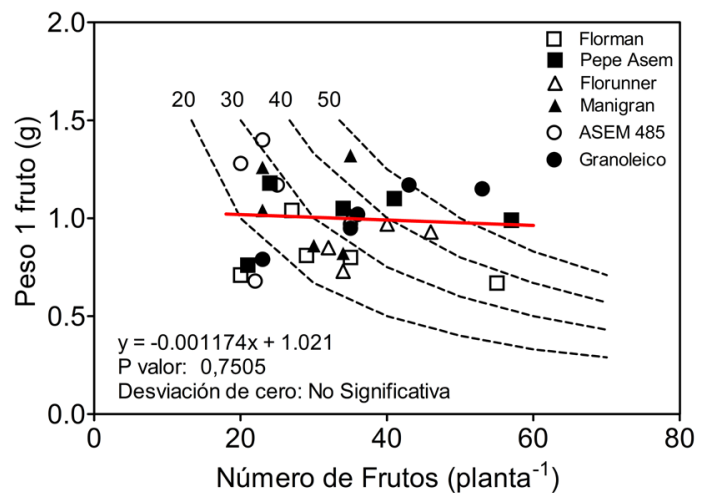

Figura 2. Peso individual de un fruto $(\mathrm{g})$ en función del número de frutos maduros por planta (frutos $\mathrm{pl}^{-1}$ ) para cultivares de tipo runner durante el ciclo experimental 2011-2012 y su regresión lineal (línea continua). Las líneas punteadas representan isocuantas de rendimiento $\left(\mathrm{g} \mathrm{pl}^{-1}\right)$

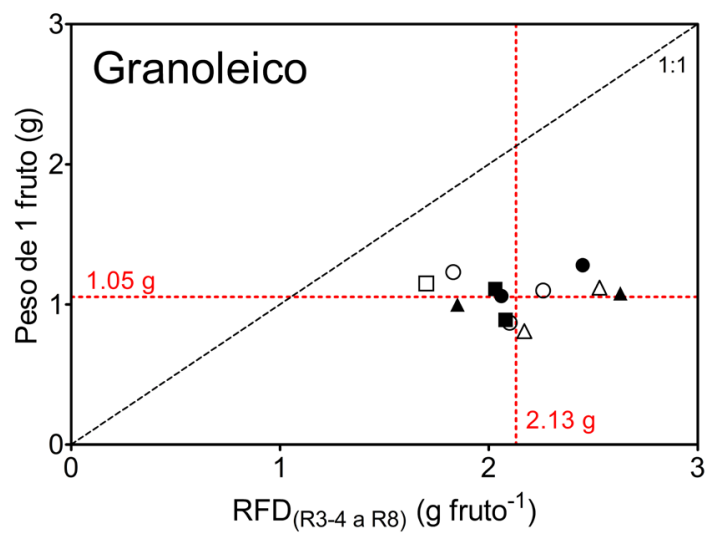

Figura 3. Peso individual de un fruto $(\mathrm{g})$ en función de la relación fuente-destino (RFD) entre R3/4 y R8 para el cultivar Granoleico sembrado en tres fechas de siembra y dos ciclos experimentales. $1^{\circ}$ FS círculos, $2^{\circ}$ FS triángulos y $3^{\circ}$ FS cuadrados. Ciclo experimental 2009-2010 símbolos vacíos; 2010-2011 símbolos llenos. Líneas punteadas indican los valores medios de cada variable. 
reproductiva) y también por aumento de su peso individual.

Contrariamente, Haro et al. (2007) hallaron diferencias en el peso de un grano según la RFD, en diferentes fechas de siembra y cultivares tipo runner. En ese trabajo se encontró que en las fechas de siembra principalmente tardías, el peso medio de un grano fue superior al aumento de biomasa total por grano, sugiriendo un uso de reservas para satisfacer la demanda durante el llenado del grano. En este sentido, Borrás, Slafer y Otegui (2004) indican una limitación por fuente en cultivos estivales durante el período de llenado de granos principalmente en zonas templadas y fechas de siembra tardías. En un estudio con diferentes intensidades de sombreo, Hang et al. (1984) encontraron limitación por fuente cuando los sombreos se aplicaron durante el período de llenado de los frutos, o sea, cuando ya existía una marcada demanda de los destinos, frutos y granos ya fijados.

En concordancia con lo descripto en este trabajo, Haro et al. (2013) mostraron una marcada limitación por destinos para siembras de la región manisera argentina realizadas en fechas normales, entre el 19/10 y 10/11, en ocho cultivares de diferente porte y patrones de ramificación desarrollados por el INTA y liberados entre los años 1948 y 2004

Una de las maneras para lograr aumentos del rendimiento sería disminuir el grado de indeterminación característico del cultivo vía el mejoramiento genético (Nautiyal et al., 2012), por empleo de biorreguladores (Senoo e Isoda, 2003) o el control de la floración (Hemsy et al., 1974). Nautiyal et al. (2012) encontraron en treinta cultivares de tipo Español una estrecha relación entre el rendimiento, la tasa fotosintética y el tamaño de los destinos, indicando una incidencia de la demanda de los destinos sobre la fotosíntesis (Gifford y Evans, 1981). Estos autores, al igual que Haro et al. (2013), remarcan que la productividad del cultivo puede ser mejorada por un aumento del tamaño de los destinos, es decir, del número de frutos fijados.

\section{CONCLUSIONES}

El rendimiento de maní tipo runner cultivado en Argentina resultó, en todos los casos y para las condiciones analizadas en este trabajo, limitado por los destinos reproductivos. No se encontraron diferencias relativas provocadas por la fecha de siembra, ciclo experimental y genotipos utilizados. Los métodos empleados en este trabajo para el análisis de la relación fuente-destino arrojaron resultados similares, validando su aplicación de manera indistinta. Estos resultados, obtenidos en un amplio rango de genotipos y condiciones ambientales indican, en concordancia con la bibliografía, la posibilidad de lograr aumentos del rendimiento a través de la mejora en el tamaño de los destinos; esto es, en la producción de estructuras y en la eficiencia reproductiva inclusive a expensas de una disminución de la capacidad de la fuente de fotoasimilados.

\section{BIBLIOGRAFÍA}

Boote, K. J. (1982). Growth stages of peanut (Arachis hypogaea L.). Peanut science, 9 (1), 35-40. https:// doi.org/10.3146/i0095-3679-9-1-11

Borrás, L., Slafer, G. A. y Otegui, M. E. (2004). Seed dry weight response to source-sink manipulations in wheat, maize and soybean: a quantitative reappraisal. Field Crops Research, 86 (2), 131-146. https://doi. org/10.1016/j.fcr.2003.08.002

Cantagallo, J. E., Medan, D. y Hall, A. J. (2004). Grain number in sunflower as affected by shading during floret growth, anthesis and grain setting. Field Crops Research, 85 (2), 191-202. https://doi.org/10.1016/ S0378-4290(03)00160-6

Cerrudo, A., Di Matteo, J., Fernandez, E., Robles, M., Pico, L. O. y Andrade, F. H. (2013). Yield components of maize as affected by short shading periods and thinning. Crop and Pasture Science, 64 (6), 580-587. https://doi.org/10.1071/CP13201

Duncan, W. G., McCloud, D. E., McGraw, R. L. y Boote, K. J. (1978). Physiological Aspects of Peanut Yield Improvement. Crop Science, 18 (6), 1015-1020. https://doi.org/10.2135/cropsci1978.0011183X00180 0060028x

Egli, D. B. y Bruening, W. P. (2001). Source-sink relationships, seed sucrose levels and seed growth rates in soybean. Annals of Botany, 88 (2), 235-242. https://doi.org/10.1006/anbo.2001.1449

Fischer, R. A. (1975). Yield Potential in a Dwarf Spring Wheat and the Effect of Shading. Crop Science, 15 (5), 607-613. https://doi.org/10.2135/cropsci1975.001 1183X001500050002x

Gambín, B. L. y Borrás, L. (2010). Resource distribution and the trade off between seed number and seed weight: a comparison across crop species. Annals of Applied Biology, 156 (1), 91-102. https://doi. org/10.1111/j.1744-7348.2009.00367.x

Gambín, B. L., Borrás, L. y Otegui, M. E. (2006). Sourcesink relations and kernel weight differences in maize temperate hybrids. Field Crops Research, 95 (2), 316- 
326. https://doi.org/10.1016/j.fcr.2005.04.002

Ghosh, A. K. y Biswas, A. K. (1995). Regulation of correlative senescence in Arachis hypogaea L. by source-sink alteration through physical and hormonal means. Journal of Agronomy and Crop Science, 175 (3), 195-202. https://doi.org/10.1111/j.1439037X.1995.tb00211.x

Giayetto, O., Morla, F. D., Fernandez, E. M., Cerioni, G. A., Kearney, M., Rosso, M. B. y Violante, M. G. (2013). Temporal analysis of branches pod production in peanut (Arachis hypogaea) genotypes with different growth habits and branching patterns. Peanut Science, 40 (1), 8-14. https://doi.org/10.3146/PS12-10.1

Gifford, R. M. y Evans, L. T. (1981). Photosynthesis, carbon partitioning, and yield. Annual Review of Plant Physiology, 32 (1), 485-509. https://doi.org/10.1146/ annurev.pp.32.060181.002413

GraphPad Prism (versión 5.00 para Windows). San Diego, USA: GraphPad Software

Hang, A. N., McCloud, D. E., Boote, K. J. y Duncan, W. G. (1984). Shade effects on growth, partitioning, and yield components of peanuts. Crop Science, 24 (1), 109-115. https://doi.org/10.2135/cropsci1984.001118 3X002400010025x

Haro, R. J., Baldessari, J. y Otegui, M. E. (2013). Genetic improvement of peanut in Argentina between 1948 and 2004: Seed yield and its components. Field Crops Research, 149. 76-86. https://doi.org/10.1016/j. fcr.2013.04.021

Haro, R. J., Dardanelli, J. L., Collino, D. J. y Otegui, M. E. (2010). Water deficit and impaired pegging effects on peanut seed yield: links with water and photosynthetically active radiation use efficiencies. Crop and Pasture Science, 61(5), 343-352.https://doi. org/10.1071/CP09234

Haro, R. J., Dardanelli, J. L., Otegui, M. E. y Collino, D. J. (2008). Seed yield determination of peanut crops under water deficit: soil strength effects on pod set, the source-sink ratio and radiation use efficiency. Field Crops Research, 109 (1), 24-33.https://doi. org/10.1016/j.fcr.2008.06.006

Haro, R. J., Otegui, M. E., Collino, D. J. y Dardanelli, J. L. (2007). Environmental effects on seed yield determination of irrigated peanut crops: Links with radiation use efficiency and crop growth rate. Field Crops Research, 103 (3), 217-228. https://doi. org/10.1016/j.fcr.2007.06.004

Hemsy, V., Rodriguez, E. L., Scandaliaris, J., Lozano Muñoz, H. y Cajal, J. A. (1974). Posibilidad de aumentar la producción de maní (Arachis hypogaea L.) controlando la floración. Revista Agronómica Noroeste Argentino, XI (4), 163-178.

Huyghe, C. (1998). Genetics and genetic modifications of plant architecture in grain legumes: a review. Agronomie, 18 (5-6), 383-411. https://doi.org/10.1051/ agro: 19980505

Morla, F. D., Giayetto, O., Cerioni, G. A. y Fernandez, E. M. (2016). Crecimiento y partición de biomasa de dos cultivares de maní (Arachis hypogaea L.) en distintas fechas de siembra en Río Cuarto, Córdoba (Argentina). European Scientific Journal, 12 (30), 334352. http://dx.doi.org/10.19044/esj.2016.v12n30p334

Nautiyal, P. C., Ravindra, V., Rathnakumar, A. L., Ajay, B. C. y Zala, P. V. (2012). Genetic variations in photosynthetic rate, pod yield and yield components in Spanish groundnut cultivars during three cropping seasons. Field Crops Research, 125, 83-91. https:// doi.org/10.1016/j.fcr.2011.08.010

Senoo, S. e Isoda, A. (2003). Effects of Paclobutrazol on Dry Matter Distribution and Yield in Peanut. Plant production science, 6 (1), 90-94. https://doi. org/10.1626/pps.6.90

Smith, C. C. y Fretwell, S. D. (1974). The optimal balance between size and number of offspring. The American Naturalist, 108 (962), 499-506. https://doi. org/10.1086/282929

Stalker, H. T. (1997). Peanut (Arachis hypogaea L.). Field Crops Research, 53 (1-3), 205-217. https://doi. org/10.1016/S0378-4290(97)00032-4

Venable, D. L. (1992). Size-number trade-offs and the variation of seed size with plant resource status. The American Naturalist, 140 (2), 287-304. https://doi. org/10.1086/285413

Williams, E. J. y Drexler, J. S. (1981). A non-destructive method for determining peanut pod maturity. Peanut Science, 8 (2), 134-141. https://doi.org/10.3146/i00953679-8-2-15

Williams, J. H. (1979). The physiology of groundnuts (Arachis hypogaea L. cv. Egret). 3. The effect of thinning at different stages of development on reproductive growth y development. Rhodesian journal of agricultural research, 17 (1), 57-62. 